\title{
Incidence of acute allergic reactions to contrast medium following CT examination on the basis of analysis of medical record data - preliminary report
}

\begin{abstract}
Introduction. A contrast-enhanced CT examination can have negative health implications. It can spark both allergic and other adverse reactions.

Aim. The study aimed at analyzing the incidence of acute allergic reactions to Ultravist 370 , used as a contrast medium in a CT examination.

Material and methods. The analysis of medical records that comprised 12295 patients who had undergone a CT examination. Women accounted for $50.7 \%$ of the study participants. Mean age of the patients was 62 years $(\min .15$; max. 92, $\mathrm{SD}=10.123$ ). In the study, 6219 patients were examined without using a contrast medium and 6076 individuals underwent a contrast-enhanced CT examination. In the further analysis, Group 1 comprised of 15 patients from the contrastenhanced CT examination group who confirmed being allergic to a contrast medium after the CT examination and Group 2 comprised 49 persons who had reported being allergic to iodine before the CT examination.

Results. 15 patients (8 women and 7 men) developed an allergy to Ultravist 370 (contrast medium). An acute allergic reaction was most likely to happen in two groups of patients: between 31-40 and 51-60 years. Some 12 out of 15 patients had no contraindications for taking an iodine-based contrast medium. The most frequent allergic reactions to Ultravist 370 were rash and urticaria (15 patients). Five patients out of those who experienced adverse reactions reported two symptoms of allergy each while other patients reported one symptom only. Patients with mild symptoms of acute reaction to a contrast medium (15 patients) received pharmacotherapy recommended by a radiologist. Some 13 individuals received Solu-Medrol intravenously and 2 patients received Solu-Medrol intravenously and Fenicort intramuscularly.

Conclusions. 1. Using Ultravist 370 in patients is safe, since there is very low risk of adverse side effects caused by the application of this contrast medium. 2. In the present study, age, gender, and risk factors contributed to the development of acute allergic reactions. However, due to the small size of the study group, it is obvious that issues like the incidence of symptoms of discomfort or intolerance of the drug need further research. 3. Owing to the fact that essential biochemical tests had not been performed on a large group of patients, doctors referring patients to a CT examination should take care of their own safety by properly preparing themselves for this procedure. 4. It seems essential to develop and implement regular trainings for CT laboratory staff with reference to the following issues: the incidence and nature of acute adverse reactions to non-ionic contrast medium (Ultravist 370), as well as the procedures for dealing with side effects.
\end{abstract}

Keywords: contrast medium, allergic reactions, medical record, computed tomography.

DOI: $10.1515 /$ pjph-2015-0034

\section{INTRODUCTION}

Contrast media are used during radiological diagnostic examinations to obtain better visualizations of the diseaseinduced changes in the patient's body. These are substances absorbing X-ray radiation to a smaller or larger degree than the surrounding tissue. On the basis of these properties, contrasting media can be divided into negative (low X-ray absorption coefficient) and positive (X-ray absorption higher than the tissue). In addition, in case of the positive ones subgroups were distinguished of contrast media soluble in water (most frequently organic iodine salts) and insoluble in water (as a rule a suspension of barium sulphate) [1-5].

A water soluble iodine agent is usually used in a CT examination is an iodine agent which dissolves in water and has urotropic properties (it is excreted by the kidneys). Examples of such agents include Uropolinum, Urografin, Ultrvist, Omnipaque, Iomeron, Hexabrid, Isovit and Visaipaque. The use of these preparations is linked to the appearance of unfavorable reactions on the part of the body. They include discomfort

\footnotetext{
${ }^{1}$ Warsaw Medical University, Poland

${ }^{2}$ Avi Diagnostic Imaging, Medical Centre, Warsaw, Poland

${ }^{3}$ Division of Public Health, Faculty of Health Science, Medical University of Warsaw, Poland

${ }^{4}$ Division of Teaching and Outcomes of Education, Faculty of Health Science, Medical University of Warsaw, Poland
} 
(the feeling of heat, metallic taste in the mouth, anxiety in the region of the heart, urgency), light symptoms (mild vomiting, urticaria, nausea, pruritus, hoarsness, cough, sneezing, profuse perspiration, feeling of heat), mild symptoms (syncope, heavy vomiting, marked urticaria, oedema of the face and the larynx, vaginal reaction, bronchial spasm) as well as heavy symptoms (pulmonary oedema, seizures, shock with a significant drop of pressure, suspension of the respiratory function, cardiac arrest) [1-5].

How to prevent this? Taking a detailed case history of every patient is essential. This allows to avoid, among others, post-contrast nephropathy, lactic acidosis, thyrotoxic crisis and even death of a patient. In spite of the fact that certain situations can be predicted, a contrast-enhanced CT causes also negative health effects in the form of side effects. They can be divided into early (developing within an hour from the administration of the contrast medium) and late (within an hour to one week from the administration). In case severe symptoms are developed, the patients should receive particular care as they are characterized by pulmonary oedema, shock with a substantial drop of pressure as well as circulatory and respiratory arrest.

\section{AIM}

The aim of the study was to analyze the frequency of the appearance of an acute post-contrast allergic reactions after the administration of Ultravist 370 used in CT examination in everyday clinical practice.

\section{MATERIAL AND METHODS}

The analysis of medical documentation covered 12295 patients of the AVI Medical Centre of Diagnostic Imaging in Warsaw, in which CT examinations were performed. Women constituted $50.7 \%$ and men $49.3 \%$ of participants of the study group. The mean age of the patients was 62 years, min. 15; max. 92, $\mathrm{SD}=10.123$ ). Some 6219 patients underwent a CT without contrast, while 6076 with contrast administration. Due to the fact that the aim of the study was to analyze the frequency of the development of acute post-contrast allergic reactions after the administration of Ultravist 370, the analysis covered 6076 patients in whom a contrast-enhanced CT was performed. From this group (6076 patients) 15 patients who confirmed allergy to the contrast medium after the examination (Group 1) and 49 patients who had reported allergy to iodine prior to the examination (Group 2) were selected for further analysis.

The CT examinations were conducted between May 2011 and January 2012. The data was collected on the basis of an analysis of medical documentation in the form of: a dedicated questionnaire completed prior to the examination as well as a form of reporting a side effect to a therapeutic product (in accordance with Annex No. 1 to the Order of the Minister of Health of 17 February 2003). All the answers from the questionnaires were analyzed and the side-effect reporting form was filled in cases where an acute postcontrast allergic reaction to the iodine contrast Ultravist 370 appeared.
Every personal questionnaire included 6 open questions and 23 closed single-choice questions. The closed questions were divided into two parts. The 17 questions included in the questionnaire focused on avoiding allergic reactions through assigning people with contraindications to the administration of a contrast medium. The second part of the questionnaire contained 6 questions aimed at the assessment of side effects following the administration of Ultravist. The questions included in the second part concerned patients who had reported such side effects.

The contrast medium was administered following the order of the radiologist monitoring the examination. Both the indications and contraindications to the administration of the contrast medium and clinical needs were regarded. Everything was done in accordance with the practice and procedures of AVI Medical Centre.

\section{RESULTS}

\section{Analysis of the content of the side effects-reporting form}

In the study group, 15 people ( 8 women and 7 men) developed an allergic reaction after receiving the Ultravist 370 contrast medium. Acute post-contrast allergic reaction was most common in the following age groups: $31-40$ and 51-60 ( 5 people in each). The analysis of data from the past allergic history showed that the majority of the patients (12 out of 15) did not have contraindications to the administration of an iodine-based contrast medium. Only 1 person reported an allergy to strawberries while 2 people did not mark an answer to this question (they could not tell whether they are allergic or not).

The most common symptoms of acute post-contrast allergic reaction appearing after the administration of Ultravis were: urticaria and rash (15 people). Some 5 people, in case of whom side effects developed, reported two allergy symptoms at one time, the remaining only one symptom each.

The patients with light symptoms of acute post-contrast reaction (15) received pharmacotherapy ordered by the radiologist. Solu-Medrol was administered to 13 patients intravenously, while Fenicort was distributed to 2 patients intramuscularly. An intra-arterial way of administration was not applied in any patient. A bolus from an automatic syringe was used in 13 patients while in 2 the contrast was administered manually from a syringe.

The analysis of the side-effects-reporting form shows that all patients with applied pharmacotherapy after an acute post-contrast allergic reaction to Ultravist 370 returned to health without any permanent consequences.

\section{Analysis of the contents of the questionnaires prior to the CT examination}

The patients were divided into two groups and the division was made on the basis of the results of questionnaires filled out by the patients prior to the examination. Group 1 comprised patients who developed allergy to the contrast medium (15 patients) while Group 2 - patients who confirmed allergy to iodine prior to the performance of the examination (49 patients). 
In Group 1, the largest number of the patients allergic to the contrast belonged to the $31-40$ or $51-60$ age groups. In Group 2, the prevailing number of patients allergic to iodine belonged to the 61-70 age group, the smallest number to the 30-40 age group. More details can be found in Table 1 .

Group 1 mostly consisted of patients weighing between 81 and $90 \mathrm{~kg}$. There were also two equally large groups of patients weighing 40-50 and 61-70 kg. On the other hand, most patients had a body mass of 61-70 kilos while those weighing 91-100 kg were the least frequent. More data can be seen in Table 2 .

In reply to the survey question: Are you allergic to iodine? - in Group 1, the prevailing majority (93\%) of the respondents chose the 'no' answer while in Group 2 all the patients reported allergy to iodine after having taken a contrast medium (other than Ultravist 370) earlier.

The following question of the questionnaire concerned the examination with an intravenous administration of an iodine contrast medium, e.g. urography. In Group 1, the prevailing majority $(80 \%)$ of the patients responded 'yes', only $20 \%$ of the patients had never had an examination with the administration of a contrast medium. All the patients from Group 2 had already been administered a contrast medium earlier.

The administration of the Ultravist contrast medium can be accompanied by a variety of unpleasant reactions. That is why the patients' answers to the question of whether the administration of the iodine contrast had been followed by discomfort symptoms, for instance, in the form of the feeling of heat, were analyzed. Details are presented in Table 3.

The next point of the questionnaire concerned the complications that might appear after the administration of the contrast medium. In Group 1, a 'no' answer was marked by $80 \%$ of the respondents, while in Group 2 some complications after the administration of the contrast medium were reported.

There was also a question about a diagnosed renal insufficiency. In Group 2, some 6\% of respondents answered 'no'. Due to the fact that renal insufficiency is counted among risk factors and is a contraindication to the administration of an iodine-based contrast medium, a determination of the creatinine concentration in blood serum was recommended (the results were normal).

The following two questions were aimed at preventing renal injury, i.e. post-contrast nephropathy, and read as follows: Was the blood serum concentration determined? and Was eGFR determined? Due to the importance of the examination, eGFR was calculated. Detailed results can be found in Table 4.

In Group 1, $6.7 \%$ of the respondents indicated the presence of nephrolythiasis, $6.7 \%$ - a renal tumor. Nephritis was not reported in any of the patients. In Group 2, nephritis was present in $12.2 \%$ of patients, a renal tumor in $10.2 \%$ and nephritis in $6 \%$. Detailed results are presented in Table 5.
TABLE 1. Frequency of allergic reactions to contrast and iodine in the study group of patients - analysis according to age.

\begin{tabular}{lcc}
\hline \hline & Age group & \% share \\
\hline \multirow{3}{*}{ Group 1 } & $20-30$ & $13.3 \%$ \\
\cline { 2 - 3 } & $31-40$ & $33.3 \%$ \\
\cline { 2 - 3 } & $41-50$ & $13.3 \%$ \\
\cline { 2 - 3 } & $51-60$ & $33.3 \%$ \\
\hline & $>60$ & $6.7 \%$ \\
\cline { 2 - 3 } Group 2 & $30-40$ & $8.2 \%$ \\
\cline { 2 - 3 } & $41-50$ & $12.2 \%$ \\
\cline { 2 - 3 } & $51-60$ & $22.4 \%$ \\
\cline { 2 - 3 } & $61-70$ & $14 \%$ \\
\cline { 2 - 3 } & $71-80$ & $18.4 \%$ \\
\hline
\end{tabular}

TABLE 2. Frequency of allergic reaction to the contrast medium and iodine in the study group - analysis of body mass dependence.

\begin{tabular}{lcc}
\hline \hline & Body mass & $\%$ share \\
\hline & $40-50$ & $13.3 \%$ \\
\cline { 2 - 3 } Group 1 & $51-60$ & $6.7 \%$ \\
\cline { 2 - 3 } & $61-70$ & $13.3 \%$ \\
\cline { 2 - 3 } & $71-80$ & - \\
\cline { 2 - 3 } & $81-90$ & $40 \%$ \\
\hline & $>90$ & $26.7 \%$ \\
\cline { 2 - 3 } Group 2 & $50-60$ & $12.2 \%$ \\
\cline { 2 - 3 } & $61-70$ & $24.5 \%$ \\
\cline { 2 - 3 } & $71-80$ & $14.3 \%$ \\
\cline { 2 - 3 } & $81-90$ & $20.4 \%$ \\
\cline { 2 - 3 } & $91-100$ & $6.1 \%$ \\
\hline
\end{tabular}

TABLE 3. Types and percentage share of discomfort symptoms after the administration of Ultravist in the two groups of patients.

\begin{tabular}{lcccc}
\hline \hline \multirow{2}{*}{$\begin{array}{c}\text { Discomfort symptoms after } \\
\text { Ultravist administration }\end{array}$} & \multicolumn{2}{c}{ Group 1 } & \multicolumn{2}{c}{ Group 2 } \\
\cline { 2 - 5 } & YES & NO & YES & NO \\
\hline Feeling of heat & $85 \%$ & $15 \%$ & $89.8 \%$ & $10.2 \%$ \\
\hline Discomfort in the heart region & $15.4 \%$ & $84.6 \%$ & $10.2 \%$ & $89.8 \%$ \\
\hline Metallic taste in the mouth & $84.6 \%$ & $15.4 \%$ & $87.8 \%$ & $12.2 \%$ \\
\hline Urgency & $85 \%$ & $15 \%$ & $91.8 \%$ & $8.2 \%$ \\
\hline
\end{tabular}

TABLE 4. Percentage share of the blood serum creatinine and eGFR in the two groups of patients.

\begin{tabular}{lcccc}
\hline \hline & \multicolumn{2}{c}{ Group 1 } & \multicolumn{2}{c}{ Group 2 } \\
\cline { 2 - 5 } & YES & NO & YES & NO \\
\hline $\begin{array}{l}\text { Blood serum creatinine } \\
\text { concentration }\end{array}$ & $93.3 \%$ & $6.7 \%$ & $85.7 \%$ & $14.3 \%$ \\
\hline eGFR level & $53.3 \%$ & $46.7 \%$ & $26.5 \%$ & $73.5 \%$ \\
\hline
\end{tabular}

TABLE 5. Percentage share of the presence of renal disease in the two study groups.

\begin{tabular}{|c|c|c|c|c|}
\hline \multirow{2}{*}{ Other renal disease } & \multicolumn{2}{|c|}{ Group 1} & \multicolumn{2}{|c|}{ Group 2} \\
\hline & YES & NO & YES & NO \\
\hline Nephrolithiasis & $6.7 \%$ & $93.3 \%$ & $12.24 \%$ & $87.76 \%$ \\
\hline Renal & $6.7 \%$ & $93.3 \%$ & $10.2 \%$ & $89.8 \%$ \\
\hline Nephritis & - & - & $6 \%$ & $94 \%$ \\
\hline
\end{tabular}


Due to the fact that certain groups of drugs taken by diabetes type II patients have to be discontinued for the examination period so that lactic acidosis would not develop, the patients were asked whether they suffer from diabetes or multiple myeloma (the latter constitutes a contra indication to contrast medium administration). The results are presented in Table 7.

Patients from both groups provided similar answers to the question about the way in which the contrast medium was administered. The data were used to evaluate whether there is any correlation between the way the contrast medium is administered and the development of an acute allergic reaction. The results can be found in Table 8 .

In part of the patients several symptoms of side effects to the administration of an iodine contrast medium were verified as shown in Table 9.

All patients from Group 2 reported allergy to iodine in the survey questionnaires. Due to the necessity of using it and having considered the benefits resulting from the examination, the radiologist decided to administer Ultravist 370 after prior pharmacological preparation (premedication). The patients received $125 \mathrm{mg}$ of Solu-Medrol intravenously. Side effects appeared in $10.2 \%$ of the patients.

Future CT examinations in these $10.2 \%$ of patients reporting side effects can be carried out only in hospital conditions, with an anesthesiologist on side. An examination with the administration of the Ultavist iodine contrast medium did not produce any permanent consequences in any of the Group 1 or Group 2 patients.

\section{DISCUSSION}

The authors conducted an analysis of scientific literature (PubMed, MEDLINE, EMBASE, SCOPUS, and looked for phrases like: iodine contrast, Ultravist 370, contrasting media in CT, contrast media, contrast reaction) allowed to find numerous publications dealing with the subject of the appearance of post-contrast allergic reactions.

The conclusion that prevails in both Polish publications and research from all around the world is that acute postcontrast allergic reactions appeared up to 1 hour after the administration of the shadowing medium. Several grades of intensity of side effect symptoms were distinguished in complications following an intravenous administration of a contrast medium (light, moderate and severe). Those symptoms were mainly of anaphylactoid etiology or resulted from the osmolar effect, for instance, direct irritation of the blood vessel wall. In spite of the well documented safety of iodine-based contrast media, side effects of varying intensity develop (from mild disturbances to fatal complications in very rare cases) [1-8].

Several post-registration observation studies and large clinical studies have been carried out in the world over the past 25 years. They assessed the application of different nonionic contrast media with the aim of identifying as well as assessing the type and frequency of appearance of side effects during radiological examinations [1].

In their study, Kopp et al. [1] found that the total frequency of appearance of acute side effects to the administration of Ultravist of $370 \mathrm{mg} \mathrm{I} / \mathrm{ml}$ concentration was comparable
TABLE 6. Percentage share of the presence of hyperthyroidism and blood serum TSH concentration in the two study groups.

\begin{tabular}{lcccc}
\hline \hline & \multicolumn{2}{c}{ Group 1 } & \multicolumn{2}{c}{ Group 2 } \\
\cline { 2 - 5 } & YES & NO & YES & NO \\
\hline Hyperthyroidism & $13.3 \%$ & $86.7 \%$ & $8.2 \%$ & $91.8 \%$ \\
\hline TSH in blood serum & $100 \%$ & - & $75.6 \%$ & $24.4 \%$ \\
\hline
\end{tabular}

TABLE 7. Frequency of the presence of diabetes and multiple myeloma in the two groups of patients.

\begin{tabular}{lcccc}
\hline \hline \multirow{2}{*}{ Comorbidities } & \multicolumn{2}{c}{ Group 1 } & \multicolumn{2}{c}{ Group 2 } \\
\cline { 2 - 5 } & YES & NO & YES & NO \\
\hline Diabetes & $26.7 \%$ & $73.3 \%$ & $30.6 \%$ & $69.4 \%$ \\
\hline Multiple myeloma & - & - & $4.1 \%$ & $95.8 \%$ \\
\hline
\end{tabular}

TABLE 8. Way of contrast administration in the two groups studied.

\begin{tabular}{lcccc}
\hline \hline \multirow{2}{*}{$\begin{array}{c}\text { Way of contrast } \\
\text { administration }\end{array}$} & \multicolumn{2}{c}{ Group 1 } & \multicolumn{2}{c}{ Group 2 } \\
\cline { 2 - 5 } & YES & NO & YES & NO \\
\hline Automatic syringe & $93 \%$ & $7 \%$ & $96 \%$ & $4 \%$ \\
\hline Manual syringe & $7 \%$ & $93 \%$ & $4 \%$ & $96 \%$ \\
\hline
\end{tabular}

TABLE 9. Types and frequency of the appearance of side effects after a contrast medium administration.

\begin{tabular}{lcc}
\hline $\begin{array}{c}\text { Side effects after iodine } \\
\text { contrast administration }\end{array}$ & Group 1 & Group 2 \\
\hline erythema/urticaria/rash & - & $20.4 \%$ \\
\hline urticaria/rash & $100 \%$ & $42.9 \%$ \\
\hline erythema/urticaria & $53.3 \%$ & $4.1 \%$ \\
\hline dyspnoea & $6.7 \%$ & $8.2 \%$ \\
\hline throat tightness & $6.7 \%$ & - \\
\hline sneezing/cough/rhinitis & $6.7 \%$ & $10.2 \%$ \\
\hline pruritus - the skin of the back & $13.3 \%$ & $4.1 \%$ \\
\hline pruritus - skin of the face & $13.3 \%$ & $6.1 \%$ \\
\hline pruritus - skin of the hands & $13.3 \%$ & $2 \%$ \\
\hline nausea/slight vomits & - & $2 \%$ \\
\hline
\end{tabular}

to the findings described in other non-ionic contrast media and amounted to $2 \%$. Unlike in the meta-analysis conducted by Caro et al. [9] who reported $0.031 \%$ severe complications, their study did not register any of them.

Prokop et al presented similar findings concerning the risk (2-4\%) of early side effects were presented [6]. Referring to the late effects, they assessed the risk of their appearance at $4-30 \%$. In consistence with the results reported by Katayama, they proved that the majority of the symptoms developing is mild or moderate $(3.1 \%)$. Severe symptoms happen in $0.4 \%$ of cases only. The frequency of very severe side effects, which can constitute a threat to life, was estimated by these authors as $0.1 \%$. By contrast, Brant et al. estimated the side-effects to a non-ionic contrasting medium at $1-3 \%[10]$.

In the $64 \mathrm{CT}$ examinations with the administration of Ultravist 370 contrast medium at AVI Medical Centre, side effects were reported in 15 patients. This constituted $23 \%$ of all the examination with a contrast medium enhancement performed.

In a multi-center, non-invasive, prospective, open observation study (without randomization), Kopp et al. looked at how often the various side effects appear, depending 
on the way of the contrast medium administration. Patients received larger doses of iopromid intra-arteriorly, in comparison to patients in whom the contrast medium was administered intravenously. More cases of side effects were recorded in the group of patients who were administered the contrast medium intravenously. Similar results were obtained in their studies by Bush et al. [11], Lasser et al. [12-14] and Shehadi [15] (conducted in the 90s). Converse results were described by Dahlstrom et al. [16], (4.3\% against $0.8 \%$ in Kopp et al.). In the study by Dahlstrom et al. [16], a significant part of the population studied were patients who had undergone brain angiography which might have affected the findings according to the authors.

The questionnaire-generated results seem to indicate that all the patients with side effects received the contrast agent intravenously. This is why these findings cannot be compared with the results obtained by Kopp et al. [1], Bush et al. [11], Lasser et al. [12-14], Sheladi [15] and Dahlstrom et. al. [16].

Another correlation analyzed in the study by Kopp et al. [1] was the way of the contrast medium administration. More side effects were reported by patients who received iopromid with an automatic syringe than by those to whom doctors administered it manually. Both Kopp et al. [1] and Miles et al. [17] suggested that extravasations which can lead to local inflammatory reactions are more common on automatic than in manual injection. This was not confirmed by Jacobs et. al. [18]. In their study they showed an absence of any link between the way of injection and the frequency of the occurrence of extravasations or any other reactions.

The results of the discussed analysis concerning the way of the contrast medium administration in AVI patients are similar to the findings of Kopp et. al. [1] as well as Miles et al. [17]. Over $90 \%$ of patients in both groups in which symptoms of acute post-contrast allergic reaction were reported, had received Ultravist 370 through an automatic syringe, a manual syringe having been used in only three of them.

The frequency of the appearance of allergic reactions depending on age was described by Petersein et al. [19], Kopp et al. [1] and Katayama et al. [20]. According to their studies, side effects were more common in patients in the 18-50 age group than in the 50-80 age group and older. The highest frequency was reported in young female patients of 18-30. Petersein et al. [19] pointed out that in young people the functioning of the immune system is better and explained the side effects in terms of a more or less efficient immune response.

The authors of the studies referred to above, as well as Pruszyński $[4,5,21]$, conclude that there is no significant correlation between the frequency of side effects and the concentration of the contrast medium and the total dose of iodine. According to Pruszyński $[4,5,21]$ these are non-anaphylactoid reactions of chemotoxic, osmotic origin which may be directly related to organic toxicity.

Acute post-contract allergic reactions were most common in Group 1, being equal in the following age groups: 30-40 and 50-60 whilst in Group 2 they affected the largest number of patients in the 60-70 age range. Unlike it is the case with the studies referred to above, in AVI Ultravist was applied solely in the 370 concentration. This means, authors have no basis grounds to comment on the theory put forward by their authors.

Both Bettmann [22] and Mertele et al. [23] as well as Kopp et. al. [1] found out that the incidence of side effects in women was much higher than in men (1.7\% against $1.3 \%)$. Lasser [12-14] observed that women of the reproductive age are at a higher risk of so-called anaphylactic reactions. Further analysis of side effects in women depending on their age revealed a higher probability of their appearance in the age group of over 70 as compared with men.

The above studies, as well as the analysis of the documentation of Group 1, indicate that women are more likely to develop acute post-contrast allergic reactions. However, as the analysed groups of AVI patients were not equal in terms of sex, the obtained Group 2 patients' results differ from the results given by Betmann [22], Mortele et al. [23] and Kopp et al. [1]. In Group 2, side effects were reported in 55.1\% of men. Because of the absence of a separate age division in women and men, it is hard to assess the results reported by the above authors.

Betmann [22] and Mortele et al. [23] stated that a higher occurrence of risk factors such as 'allergic defect' in the women taking part in the study need not necessarily be treated as an indicator of an increased risk. In the study by Kopp et al. [1], 62\% of allergic patients were women. The authors estimated that the incidence of side effects was higher in patients with 'allergic defect' than in patients reporting prior reactions after the administration of a contrast medium (an increase in risk at a level of 3.4 and 6.2 times, respectively). In both groups, the frequency remained on a relatively low level and hence it was concluded that side effects do not develop within the mechanism of classic allergy.

The authors' own research shows that all Group 2 patients confirmed allergy to iodine appearing in contrasts. Side effects after a subsequent administration occurred in $10.2 \%$ of the patients only. In this group, some $53 \%$ of the patients reported having an 'allergic defect' which stands for an allergy to drugs, food and chemicals. That is why the findings of Betmann and Mortele et al. are comparable with the findings resulting from an analysis of the medical documentation of AVI patients. According to Pruszyński, the frequency of the occurrence of side effects is higher in patients allergic to other pharmacological preparations. The above data served to assess the appearance of side effects depending on risk factors.

The study carried out by Narita et al. [24] revealed that the incidence of bronchial asthma and other allergic diseases that has been growing recently can be linked to the appearance of estrogens in the environment. These authors suggest that this confirms the hypothesis that both reactions after the administration of contrast media, allergy and anaphylaxis occur more frequently in the presence of high estrogen concentrations.

As the present analysis did not cover an analysis of the influence of the concentration of estrogens on the incidence of bronchial asthma, reaction to contrast media, allergy and anaphylaxis, it is difficult to comment on the opinion presented by the authors of the studies referred to above. In reply to a question about a possible past history of bronchial asthma, the majority (73.3\%) of Group 1 patients 
and Group 2 patients (71.4\%) who reported side effects, chose the 'no' answer. The question whether bronchial asthma affects the development of an acute allergic reaction was found to be significant. Using the studies that had been performed to date $[4,5,21]$ Pruszyński stated that bronchial asthma belongs to the group of diseases in which the intravascular administration of a contrast medium is linked to a particular risk of complications.

From among the 74717 patients of the whole population taking part in the prospective study by Kopp et al. [1], 19402 people were qualified to the risk group with major comorbidities. In these groups, the highest frequency (2\%) of side effects was reported.

The study by Pruszyński, Prokop and Galanski [4-6,21] shows that apart from bronchial asthma presented above, also other diseases were included in the group of significant comorbidities. They were: renal insufficiency, hyperthyroidism, heart diseases and neurological diseases. They can contribute to a higher incidence of side effects and that is why the patients' answers are seriously taken into account when Ultravist 370 contrast medium is to be administered. In the majority of AVI patients these diseases were rarely present.

The analysis presented in this study also concerned symptoms of discomfort (feeling of heat, metallic taste in the mouth as well as urgency). Kopp et al. described them as 'indicators of tolerance' present in some $0.5 \%$ of patients. Conversely, as Prokop, Galanski [6] and Pruszyński [4,5,21] show in their findings, they are present in $45 \%$ of cases.

The presented results of AVI patients with symptoms of discomfort during routine CT examinations are significantly higher and the number can be estimated as about $85 \%$.

Our own results concerning the types of symptoms of side effects confirm the opinions presented in the publications by Pruszyński [4,5,21], Prokop and Galanski [6]. Those two researchers claim that skin symptoms, like urticaria, are anaphylactoid reactions. It results from an amplified local cutaneous blood flow and usually develops in conjunction with pruritus. The regions most commonly affected include the face, the neck and the chest. Symptoms on the part of the respiratory system in the form of oedema of the air passages and the larynx can be accompanied by throat tightness and cause anxiety in the majority of patients. On the other hand, dyspnoea, one of the most important symptoms, can develop in patients with a congestive heart failure. This is what justifies their presence in the questionnaire. The authors of this work found out that over $90 \%$ of patients in both groups did not report the occurrence of dyspnoea.

Based on the authors' own research as well as literature review, it seems that acute side effects after taking Ultravist 370 occur very rarely. Due to the fact that CT has become a very popular examination over the past ten years, few studies into the development of acute allergic reactions have been carried out and hence a recommendation that they deserve being continued. What comes to be an indispensible action on the part of medical personnel is to raise patients' awareness of possible threats associated with the performance of a CT. It needs emphasizing that this examination is not neutral to children and women of the reproductive age. Any referrals for CT examinations should be well thought over as more and more infants and small children are referred for CT examinations with a contrast medium. It is also well justi- fied that side effects should be honestly reported by CT lab employees and only then will the data be more reliable than the results of clinical studies alone.

\section{CONCLUSIONS}

1. It is safe to use Ultravist 370 , as the probability of potential side effects is very low.

2. In the present study, characteristics like age, gender, and other factors contributed to the development of acute allergic reactions. However, due to the small size of the study group, it is recommended that the study of the incidence of symptoms of discomfort or intolerance of the drug be continued.

3. Owing to the fact that the essential biochemical tests had not been performed on a large group of patients, doctors referring patients for a CT examination should take care of their safety by properly preparing them for this procedure.

4. Development and implementation of regular trainings for CT laboratory staff with reference to the following issues: the incidence and nature of acute adverse reactions to non-ionic contrast medium (Ultravist 370) as well as the procedures for dealing with side effects is needed.

\section{REFERENCES}

1. Kopp AF, Mortelle KJ. Częstość występowania ostrych reakcji po lopromidzie: badanie obserwacyjne 74717 chorych po wprowadzeniu preparatu na rynek. Acta Radiol. 2008;49(8):45-9.

2. Wysocka A, Wysocki H. Wybrane działania niepożądane środków kontrastowych stosowanych u chorych poddanych przezskórnym interwencjom na naczyniach wieńcowych, Now Lek. 2007;76(2):121-5.

3. Koziński M, Kubica MJ. Wpływ rodzaju i objętości wybranych niejonowych środków cieniujących na odpowiedź zapalną u pacjentów poddanych angioplastyce wieńcowej. Folia Cardiol. 2005;11(12):74051.

4. Pruszyński B. Radiologia. Diagnostyka Obrazowa Rtg, TK, USG, MR i medycyna nuklearna. Warszawa: Wyd. PZWL; 2003. p. 15-20, 51-3, 66-8, 77-80, 90-2.

5. Pruszyński B. Diagnostyka Obrazowa. Podstawy teoretyczne i metodyka badań. Warszawa: PZWL; 2000. p. 18, 22-6, 66-9, 79, 143-6, 408-17.

6. Prokop M, Galanski M. Spiralna i wielorzędowa tomografia komputerowa człowieka. Warszawa: Medipage; 2007. p. 2-3, 9-11, 18-20, 86-90, 102.

7. Baert AL, Knauth M. Medical Radiology. Diagnostic Imaging. Berlin: Contrast Media, Springer-Werlag; 2006/2009.

8. Morcos SK. Acuta serious and fatal reactions to contrast media: our current understanding; BJR. 2005;78:686-93.

9. Caro JJ, Trindade E, McGregor M. The risks of heath and of severe nonfatal reactions with high - vs low osmo-lalit contrast media: a metaanalysis. Am J Roentgenol. 1991;156:825-32.

10. Brant WE, Helms C. Podstawy diagnostyki radiologicznej. Warszawa: Medipage; 2006. p. 9-11, 48, 26-8.

11. Bush WH, Swanson DP.Acute reactions to intravascular contrast media: types, risk factors, recognition, and specific treatment. Am J Roentgenol. 1991; 157:1153-61.

12. Lasser EC, Berry C. Pretreatment with corticosteroids to prevent adverse reaction to monionic contrast media. Am J Roentgenol. 2012;98(5):18996.

13. Lasser EC. Contrast reactions. Eked data from contrastreaction surveys. Invest Radiol. 1990;25(Suppl. 1):145.

14. Lasser EC. contrast reaction [http://www.google.pl/search?g=lasser++contrast +reaction]

15. Shehadi WH. Contrast media adverse reactins: occurrence, reccurence, and distribution patterns. Radiol. 1982;143:117-9. 
16. Dahlstrom K, Shaw DD, Clauss W, et al. Summary of U.S. and European intravascular experience with iohexol based on the clinical trial program. Invest Radiol. 1985;20(Suppl. 1):117-21.

17. Miles SG, Rasmussen JF, Litwiller T, et al. Safe use of an intravenous power injector for CT; experience and protocol. Radiol. 1998;209:411-6.

18. Jacobs JE, Birnbaum BA, Longlotz CP. Contrast media reactions and extravasation; relationship to intravenous injection rates. Radiol. 1998;209:411-6.

19. Petersein J, Peters CR, Wolf M, Hamm B. Results of the safety and efficacy of iobitridol in more than 61,000 patients. Eur Radiol. 2003;13: 2006-11.

20. Katayama H, Yamaguchi K, Tozuka T, et al. Adverse reactions to ionic and nonionic contrast media. A reports from the Japanese Committee on the Safety of Contrast Media. Radiol. 1990;175:621-8.

21. Pruszyński B. Wskazania do badań obrazowych. Warszawa: Wydawnictwo Lekarskie PZWL; 2011. p.88-91, 186-7, 190-5.

22. Bettman MA. Freguenthyasked guesstions iodinated contrast agents. Radiographics. 2004;24(Suppl. 1):310.

23. Mortele KJ, Oliva MR, Ondatequi S, et al. Universal USC of nonionic iodinated kontrast medium for CT: evaluation of safety in a large urban teaching hospital. Am J Rpentgenol. 2005;184:314.

24. Narita S, Goldblum RM, Watson CS, et al. Environmental estrogens induce mast cell degranulation and enhance IgE-mediated release of allergic mediators. Environ Health Perspect. 2007;115:485-2.

\section{Corresponding author}

Dr hab. n o zdr. Joanna Gotlib

81 Żwirki i Wigury Str., 02-091 Warszawa

tel. (22) 57-20-490; fax. (22) 57-20-491

E-mail: joanna.gotlib@wum.edu.pl 